\title{
Association of critical illness scores (q-SOFA and APACHE) and multimorbidity in patients admitted to internal medicine step down units
}

\author{
Authors: Sher Sethi, ${ }^{\mathrm{A}}$ Madiha Iqbal, ${ }^{\mathrm{A}}$ Amber Sabeen, ${ }^{\mathrm{A}}$ Sania Sabir ${ }^{\mathrm{A}}$ and Aysha Almas ${ }^{\mathrm{A}}$
}

\section{Introduction}

A large number of patients worldwide have multimorbidity (two or more chronic diseases). In the past few decades, the prevalence of multimorbidity has been rising steeply and managing such patients is a challenge. ${ }^{1}$ The majority of these patients are admitted to step down units or SDUs (rather than intensive care units). Mortality among these patients is around $10 \%$, so it is important to assess the severity of illness to guide prognosis. There are various scoring systems developed in this context to calculate the severity of illness. ${ }^{2}$ These scoring systems aim to help physicians estimate disease severity, identify possible outcomes and prepare them for the appropriate management. ${ }^{3}$ We aimed to determine the level of illness by using two different scoring systems: the quick Sequential Organ Failure Assessment Score (q-SOFA) and Acute Physiology and Chronic Health Evaluation (APACHE).

\section{Methods}

We carried out a cross-sectional analytical study which included adult patients aged $>18$ years admitted to SDUs at the Aga Khan Hospital in 2016. Data were acquired for a sample of $1,191 / 3,500$ patient records, and data for $1,002 / 1,191$ patients were included in the study. Data on demographics, comorbid conditions, admitting diagnosis, laboratory investigations, mortality and readmission to SCU from the ward/emergency room was recorded. APACHE II was used, comprising 12 physiological variables, age and chronic health evaluation. Q-SOFA utilises blood pressure, respiratory rate and the Glasgow Coma Scale (GCS) to calculate the severity of critical illness.

\section{Results}

The study included 1,002 patients with a mean (standard deviation (SD)) age of 62.2 (16.5) years; $493(49.2 \%)$ were men and $923(92.1 \%)$ were full-code (ie they had given permission for resuscitation, intubation and ventilator support). These 1,002 patients had major comorbidities and included 549

Authors: ${ }^{\mathrm{A}}$ Aga Khan University Hospital, Karachi, Pakistan

\begin{tabular}{|c|c|c|c|}
\hline \multirow[t]{2}{*}{ Diagnosis } & \multirow{2}{*}{$n=1,002(\%)$} & q-SOFA & APACHE II \\
\hline & & Mean $\pm S D$ & Mean $\pm S D$ \\
\hline $\begin{array}{l}\text { Acute kidney injury } \\
\text { or chronic kidney } \\
\text { disease }\end{array}$ & $399(39.8 \%)$ & $1.8 \pm 0.6$ & $14.7 \pm 4.8$ \\
\hline Pneumonia & $291(29.0 \%)$ & $1.8 \pm 0.6$ & $12.7 \pm 5.1$ \\
\hline $\begin{array}{l}\text { Urinary tract } \\
\text { infection }\end{array}$ & $211(21.1 \%)$ & $1.8 \pm 0.6$ & $13.4 \pm 5.1$ \\
\hline $\begin{array}{l}\text { Pulmonary oedema / } \\
\text { heart failure }\end{array}$ & $171(17.1 \%)$ & $1.7 \pm 0.6$ & $14.2 \pm 5.2$ \\
\hline Sepsis / septic shock & $159(15.9 \%)$ & $1.9 \pm 0.6$ & $13.6 \pm 5.2$ \\
\hline
\end{tabular}

(54.8\%) with diabetes, $708(70.7 \%)$ with hypertension, 282 (28.3\%) with ischaemic heart disease (IHD), 109 (10.9\%) with chronic obstructive pulmonary disease (COPD) / asthma and $263(26.2 \%)$ with chronic kidney disease.

Multimorbidity was found in 888 patients (88.8\%). Comparison of q-SOFA with multimorbidity showed a mean score of 1.8 with SD of 0.6 in multimorbid patients, with a mean score of 1.8 with
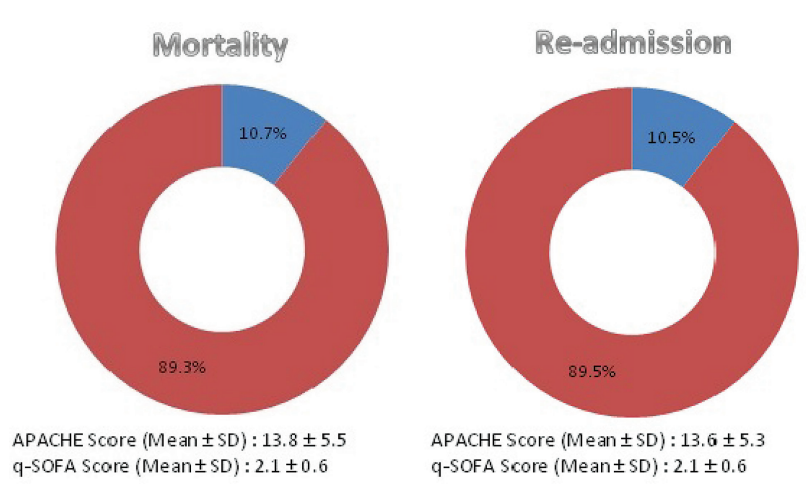

Fig 1. Mortality and readmission scores using APACHE and q-SOFA. 
SD of 0.6 in non-multimorbid patients $(\mathrm{p}=0.940)$. Comparisons of APACHE II with multimorbidity showed a mean score of 13.1 with SD of 5.1 in multimorbid patients, with a mean score of 7.4 with $\mathrm{SD}$ of 4.7 in non-multimorbid patients (p-value 0.000 ). See Table 1 and Fig 1.

\section{Conclusion}

APACHE had a direct association with multimorbidity when compared with q-SOFA. Patients with multimorbidity had a high APACHE score, indicating that they were at higher risk of mortality. We conclude that APACHE is a better predictor of illness in patients admitted to SDUs.

\section{Conflicts of interest}

None declared.

\section{References}

1 World Health Organization. Global status report on noncommunicable diseases 2010. Geneva: WHO, 2011. www.who.int/nmh/ publications/ncd_report2010/en/ [Accessed 7 February 2020].

2 Innocenti F, Tozzi C, Donnini C et al. SOFA score in septic patients: incremental prognostic value over age, comorbidities, and parameters of sepsis severity. Intern Emerg Med 2017;13:405-12.

3 Ridley S. Severity of illness scoring systems and performance appraisal. Anaesthesia 1998;53:1185-94. 